\title{
Superfluid characteristics of the attractive Hubbard model for various lattice structures
}

\author{
W. R. Czart, T. Kostyrko \& S. Robaszkiewicz \\ Institute of Physics, A.Mickiewicz University, Umultowska 85, 61-614 Poznań, POLAND.
}

\begin{abstract}
We study the basic thermodynamic and electromagnetic properties of the superconductor described by the negative $-U$ Hubbard model ( gap parameter $\Delta$, critical temperature $T_{\mathrm{C}}$, London penetration depth $\lambda$, thermodynamic critical field $H_{\mathrm{C}}$ and Ginzburg-Landau correlation length $\left.\xi_{\mathrm{G}-\mathrm{L}}\right)$.

The calculations are performed for square $(\mathrm{SQ}, \mathrm{d}=2)$, simple cubic $(\mathrm{SC}, \mathrm{d}=3)$ and face-centered cubic $(\mathrm{FCC}, \mathrm{d}=3$ ) lattices. We analyze the results as a function of electron density $n$ and interaction $U$ using the Hartree-Fock approximation in the weak-to-intermediate $U$ case combined with the conclusions from the perturbation theory valid in the strong coupling limit. From the equality: $\lambda / \xi_{\mathrm{G}-\mathrm{L}}=1 / \sqrt{2}$ we find the boundaries between local and nonlocal electronic behaviour of the $U<0$ Hubbard model in the $U-n$ parameter space. Using the calculated values of $T_{\mathrm{C}}$ and $\lambda$ we compare the 'universal plot' of $T_{\mathrm{C}} / T_{\mathrm{C}}^{\mathrm{m}}$ vs. $\left[\lambda(0) / \lambda^{\mathrm{m}}(0)\right]^{2}$ ( where: $T_{\mathrm{C}}^{\mathrm{m}}$ denotes maximum $T_{\mathrm{C}}$ as a function of $n$ and $\lambda^{\mathrm{m}}(0)$ corresponds to $\left.T_{\mathrm{C}}^{\mathrm{m}}\right)$ with the recent experimental data for various families of nonconventional superconductors and conclude that the best agreement can be obtained for intermediate values of the local attraction.
\end{abstract}

Keywords: negative- $U$ Hubbard model, London penetration depth, high- $T_{\mathrm{C}}$ superconductivity 
PACS numbers: 74.30, 74.20.-z, 71.28.+d, 74.25.Ha 


\section{INTRODUCTION}

Among the recently studied superconducting compounds there are several groups of materials (copper oxides, fullerides, Chevrel phases, barium bismuthates ...) which share some nonconventional features like high $T_{\mathrm{C}}$ with relatively low carrier concentration, short coherence length together with extremely small $\xi_{\mathrm{G}-\mathrm{L}} / \lambda$ ratio and universal dependence of $T_{\mathrm{C}} / T_{\mathrm{C}}^{\mathrm{m}}$ vs. $\left[\lambda(0) / \lambda^{\mathrm{m}}(0)\right]^{2}$ [四,2]. Above features suggest that a short range almost nonretarded attraction is responsible for pairing in these systems [3]. One of the simplest effective models to describe this situation is the negative- $U$ Hubbard hamiltonian. This model constitutes a common basis for the description of the superconductors with weak local electron pairing, being in many ways similar to the classical BCS systems, and superconductors with strong attraction where the local pairs conform to weakly charged hard core bosons [3]. It has been considered as an effective model of superconductivity in the family of cuprates [4, 5.,6], the barium bismuthates [7],8], the fullerides [9, 10, 11, 12, 13] and the Chevrel phases [3].

In our paper we present some new results of an analysis of the several basic thermodynamic and electromagnetic properties of this model for various types of lattice structure. We have concentrated on the case of (i) square (SQ, $d=2$ ), (ii) simple cubic (SC, $d=3$ ) and (iii) face-centered cubic (FCC, $d=3$ ) lattices, i.e. structures representing (i) the family of cuprates, (ii) the Chevrel phases and barium bismuthates and (iii) the fullerides, respectively. For comparison we also present the results obtained for a model rectangular density of states $\mathcal{D}(\varepsilon)$. To calculate quantities of interest we use the (broken symmetry) HartreeFock approximation (HFA) which is known to give credible results at $T=0 \mathrm{~K}$ as concerns energy of the ground state, the energy gap, the chemical potential as well as the collective excitations in the whole interaction range, interpolating smoothly between the weak and strong- $U$ limit, where it matches the results of the perturbation theory developed from the zero-bandwidth limit [14]. On the other hand the HFA leads to qualitatively erroneous results for the the thermodynamic critical field $H_{\mathrm{C}}$ and Ginzburg-Landau correlation length 
$\xi_{\mathrm{G}-\mathrm{L}}$ in the strong attraction limit. This failure is due to the fact that the HFA greatly overestimates the energy of the normal state which is used in a standard calculation of $H_{\mathrm{C}}$ and next $\xi_{\mathrm{G}-\mathrm{L}}$. In order to discuss the behaviour of $H_{\mathrm{C}}, \xi_{\mathrm{G}-\mathrm{L}}$ and Ginzburg ratio $\kappa=\frac{\lambda}{\xi}$ in the whole interaction range we have completed the results of the HFA calculation with the ones of the perturbation theory. We will also point out a possible relevance of our results to the recent experimental data for the short-coherence length superconductors.

\section{GENERAL FORMULATION}

We consider the negative- $U$ Hubbard Hamiltonian in the hypercubic lattice

$$
H=t \sum_{\vec{m} \vec{\delta} \sigma}\left(\mathrm{e}^{i \Phi_{m m+\delta}} c_{\vec{m} \sigma}^{+} c_{\vec{m}+\vec{\delta} \sigma}+\text { H.c. }\right)-|U| \sum_{\vec{m}} n_{\vec{m} \uparrow} n_{\vec{m} \downarrow}
$$

where $t$ is the hopping integral, $\vec{\delta}$ is a unit lattice vector and the exponential (Peierls) factors in (11) account for the coupling of electrons to the magnetic field via its vector potential $\vec{A}(\vec{r})$. $\Phi_{m m+\delta}=-\frac{e}{\hbar c} \vec{A}(\vec{m}) \vec{\delta}$, and $e$ is the electron charge.

For weak vector potential the expectation value of the Fourier transform of the total current operator in $\alpha$ direction $(\alpha=x, y, z)$ can be obtained from the linear response theory [16,17] as

$$
J_{\alpha}(\vec{q}, \omega)=N \frac{c}{4 \pi} \sum_{\beta}\left[\delta_{\alpha \beta} K^{\text {dia }}+K_{\alpha \beta}^{\text {para }}(\vec{q}, \omega)\right] A_{\beta}(\vec{q}, \omega)
$$

Here we have separated the total response kernel $K_{\alpha \beta}(\vec{q}, \omega)$ into a diamagnetic part and a paramagnetic part. The diamagnetic contribution evaluated within HFA is

$$
\begin{aligned}
K^{d i a}= & \frac{8 \pi e^{2}|t|}{\hbar^{2} c^{2} a_{\perp}} \frac{1}{N} \sum_{\vec{k} \sigma}<c_{\vec{k} \sigma}^{+} c_{\vec{k} \sigma}>\cos k_{\alpha}= \\
& =\frac{8 \pi e^{2}|t|}{\hbar^{2} c^{2} a_{\perp}} \frac{1}{N} \sum_{\vec{k}}\left[1-\frac{\lambda_{\vec{k}}}{E_{\vec{k}}} \tanh \left(\beta E_{\vec{k}} / 2\right)\right] \cos k_{\alpha}
\end{aligned}
$$

where $\lambda_{\vec{k}}=\epsilon_{\vec{k}}-\bar{\mu}, \epsilon_{\vec{k}}=t \sum_{\vec{\delta}} \mathrm{e}^{i \vec{k} \vec{\delta}}$ and $E_{\vec{k}}=\sqrt{\lambda_{\vec{k}}^{2}+\Delta^{2}}$ is the quasiparticle energy with the superconducting gap parameter 
$\Delta=|U| x_{0}=\frac{|U|}{N} \sum_{i}\left\langle c_{i \uparrow}^{+} c_{i \downarrow}^{+}\right\rangle=\frac{|U|}{N} \sum_{\vec{k}}\left\langle c_{\vec{k} \uparrow}^{+} c_{-\vec{k} \downarrow}^{+}\right\rangle$. The chemical potential is $\mu=\bar{\mu}-|U| n / 2$, $N$ and $n=\frac{N_{e}}{N}$ denote the number of lattice sites and the number of electrons per site, respectively and $0<n<2$. The values of $\Delta$ and $\mu$ are obtained as a solution of the system of the selfconsistent equations [15]:

$$
\begin{aligned}
\Delta & =\frac{|U| \Delta}{2 N} \sum_{\vec{k}} \tanh \left(\beta E_{\vec{k}} / 2\right) / E_{\vec{k}}, \\
n & =\frac{1}{N} \sum_{\vec{k}}\left[1-\lambda_{\vec{k}} \tanh \left(\beta E_{\vec{k}} / 2\right) / E_{\vec{k}}\right],
\end{aligned}
$$

whereas the free energy per site of the superconducting (SS) phase and the normal (NO) phase is given by:

$$
\begin{array}{r}
\frac{F^{\mathrm{SS}}}{N}=\bar{\mu}_{\mathrm{SS}}(n-1)+\frac{\Delta^{2}}{|U|}-\frac{|U|}{4} n^{2}-\frac{2}{\beta N} \sum_{\vec{k}} \ln 2 \cosh \frac{\beta E_{\vec{k}}}{2}, \\
\frac{F^{\mathrm{NO}}}{N}=\bar{\mu}_{\mathrm{NO}}(n-1)-\frac{|U|}{4} n^{2}-\frac{2}{\beta N} \sum_{\vec{k}} \ln 2 \cosh \frac{\beta \lambda_{\vec{k}}}{2},
\end{array}
$$

where $\bar{\mu}_{\mathrm{NO}}$ is determined from:

$$
n=\frac{1}{N} \sum_{\vec{k}}\left(1-\tanh \frac{\beta \lambda_{\vec{k}}}{2}\right)
$$

The expression for the paramagnetic part of the kernel reads

$$
\begin{aligned}
& K_{\alpha \alpha^{\prime \prime}}^{\text {para }}(\vec{q}, \omega)=-\frac{1}{N} \frac{16 \pi e^{2} t^{2}}{\hbar^{2} c^{2}} \sum_{\vec{k}} \sin \vec{k}_{\alpha} \sin \vec{k}_{\alpha^{\prime \prime}}[ \\
& \left(1+\frac{\lambda_{\vec{k}} \lambda_{\vec{k}-\vec{q}}+\Delta^{2}}{E_{\vec{k}} E_{\vec{k}-\vec{q}}}\right)\left[n_{F}\left(E_{\vec{k}}\right)-n_{F}\left(E_{\vec{k}-\vec{q}}\right)\right] \times \\
& \times\left(\frac{1}{\omega-\left(E_{\vec{k}-\vec{q}}-E_{\vec{k}}\right)+i \varepsilon}-\frac{1}{\omega+E_{\vec{k}-\vec{q}}-E_{\vec{k}}+i \varepsilon}\right)+ \\
& +\left(1-\frac{\lambda_{\vec{k}} \lambda_{\vec{k}-\vec{q}}+\Delta^{2}}{E_{\vec{k}} E_{\vec{k}-\vec{q}}}\right)\left[1-n_{F}\left(E_{\vec{k}}\right)-n_{F}\left(E_{\vec{k}-\vec{q}}\right)\right] \times \\
& \left.\times\left(\frac{1}{\omega-\left(E_{\vec{k}-\vec{q}}+E_{\vec{k}}\right)+i \varepsilon}-\frac{1}{\omega+E_{\vec{k}}+E_{\vec{k}-\vec{q}}+i \varepsilon}\right)\right] .
\end{aligned}
$$

In the London superconductors the magnetic field penetration depth $\lambda(T)$ is determined by the sum of diamagnetic and paramagnetic part of the total kernel in the static limit $\lambda(T)=\lim _{q_{y} \rightarrow 0}\left[-K^{\text {dia }}-K_{x x}^{\text {para }}\left(q_{x}=0, q_{y}, q_{z}=0, \omega=0\right)\right]^{-1 / 2}$ [16,17]. 
At $T=0 \mathrm{~K}$ the paramagnetic part of the kernel becomes important in determining $\lambda$ when the correlation length becomes greater than the penetration depth and we deal in this case with nonlocal (Pippard) superconductor. This situation is common in many low$T_{\mathrm{C}}$ systems. The short-coherence length materials, including the high $T_{\mathrm{C}}$ superconductors represent the opposite, i.e. the London limit. In the latter case the ground state penetration depth is determined entirely by the $\vec{q} \rightarrow 0$ limit of the kernel where the paramagnetic part of the kernel vanishes and $\lambda$ is given by:

$$
\lambda=\frac{1}{\sqrt{-K^{d i a}}}
$$

In our calculation of $\lambda$ we restrict ourselves to the London limit having in mind the properties of the systems of interest and finaly determine the area in the $U-n$ parameters space where the local approximation may be valid. The value of the penetration depth calculated in this way is qualitatively good both in the weak and strong- $U$ limits, in the latter case approaching the results of the perturbation theory, as will be shown below.

Using the value of the penetration depth and the difference of the free energy between normal and superconducting phase one is able to determine the thermodynamic critical field $H_{\mathrm{C}}$ and the Ginzburg-Landau correlation length $\xi_{\mathrm{G}-\mathrm{L}}$ as

$$
\begin{aligned}
\frac{H_{\mathrm{C}}^{2}(T)}{8 \pi} & =\frac{F^{\mathrm{NO}}(T)-F^{\mathrm{SS}}(T)}{N a_{\perp} a_{\|}^{2}}, \\
\xi_{\mathrm{G}-\mathrm{L}} & =\frac{\Phi_{0}}{2 \pi \sqrt{2} \lambda H_{\mathrm{C}}}
\end{aligned}
$$

where $\Phi_{0}=\frac{h c}{2 e}$, and to obtain the estimations for the critical fields $H_{\mathrm{c} 1} \simeq \frac{\ln \kappa}{\kappa} H_{\mathrm{C}} \sim \frac{\ln \kappa}{\lambda^{2}}$ and $H_{\mathrm{c} 2} \simeq \frac{\Phi_{0}}{2 \pi \xi_{\mathrm{G}-\mathrm{L}}^{2}}$, where $\kappa=\frac{\lambda}{\xi_{\mathrm{G}-\mathrm{L}}}$. At $T=0, \frac{F(0)}{N}=E_{0}=\frac{1}{N}\langle H\rangle_{(T=0)}$. Within HFA the expressions determining $E_{0}^{\mathrm{NO}}$ and $E_{0}^{\mathrm{SS}}$ are given by:

$$
\begin{gathered}
E_{0}^{\mathrm{NO}}=\frac{F^{\mathrm{NO}}(0)}{N}=E_{\mathrm{NO}}^{\mathrm{K}}(0)-\frac{1}{4}|U| n^{2}, \\
E_{0}^{\mathrm{SS}}=\frac{F^{\mathrm{SS}}(0)}{N}=E_{\mathrm{SS}}^{\mathrm{K}}(0)-\frac{\Delta^{2}}{|U|}-\frac{1}{4}|U| n^{2}
\end{gathered}
$$

where 


$$
E_{\mathrm{NO}(\mathrm{SS})}^{\mathrm{K}}=\frac{1}{N} \sum_{\vec{k} \sigma} \epsilon_{\vec{k}}\left\langle c_{\vec{k} \sigma}^{+} c_{\vec{k} \sigma}\right\rangle_{\mathrm{NO}(\mathrm{SS})}
$$

is the average value of the kinetic energy term in the $\mathrm{NO}(\mathrm{SS})$ phase.

The HFA calculation of the energy of the ordered state at $T=0 \mathrm{~K}$ are reliable for any $U$ [3] and $E_{0}^{\mathrm{SS}}$ reduces correctly to the exact value: $U n / 2$ in the zero bandwidth limit where the electrons form a system of on-site pairs and singly occupancy of sites is prohibited. On the other hand the HFA energy of the normal phase in this limit is equal to $U n / 4$ instead of $U n / 2$ thereby producing the incorrect energy difference of $U n / 4$. In order to remedy this inconsistency, at least in part, we resort to the results of the perturbation theory in the calculation of $E_{0}^{\mathrm{NO}}$ for $|U| \gg t$. The hamiltonian (雨) reduces in this case to the pseudospin-1/2 Heisenberg model working in the subspace of states with no singly occupied sites [3, 18, 19]: 


$$
\begin{aligned}
\tilde{\mathrm{H}}= & -\frac{|U|}{2} \sum_{\vec{j}}\left(2 \rho_{\vec{j}}^{z}+1\right)-\frac{1}{2} \sum_{\vec{j}, \vec{\delta}} J_{\vec{\delta}}\left(\rho_{\vec{j}}^{+} \rho_{\vec{j}+\vec{\delta}}^{-} \mathrm{e}^{-2 i \frac{e}{\hbar c} \vec{A}(\vec{j}) \vec{\delta}}+\text { H.c. }\right) \\
& +\sum_{\vec{j}, \vec{\delta}} J_{\vec{\delta}} \rho_{\vec{j}}^{z} \rho_{\vec{j}+\vec{\delta}}^{z}-\frac{N}{4} Z J,
\end{aligned}
$$

where $2 \rho_{i}^{z}=\left(n_{i \uparrow}+n_{i \downarrow}-1\right), \rho_{i}^{+}=c_{i \uparrow}^{+} c_{i \downarrow}^{+}, J_{\vec{\delta}}=2 t^{2} /|U|$ and $Z$ denotes a number of nearest

neighbours. The hard-core boson operators: $\rho_{\vec{j}}^{ \pm}, \rho_{\vec{j}}^{z}$ satisfy the commutation rules of the $s=\frac{1}{2}$ operators. The electron number condition is:

$$
n=\frac{1}{N} \sum_{\vec{j}}\left\langle 2 \rho_{\vec{j}}^{z}+1\right\rangle
$$

For $x_{0}=\left\langle\rho^{+}\right\rangle=\frac{1}{N} \sum_{i}\left\langle\rho_{i}^{+}\right\rangle \neq 0$ the hamiltonian (16) describes the superconducting state and the MFA result for the $E_{0}^{\mathrm{SS}}$ is equal to the corresponding HFA one up to terms of order $t^{2} /|U|$ inclusive (see Sec.3). The MFA calculation of the ground state energy for the normal state, $x_{0}=0$, gives:

$$
\bar{E}_{0}^{\mathrm{NO}}=\frac{\langle\tilde{\mathrm{H}}\rangle}{N}=-\frac{1}{2}|U| n+\frac{1}{4} Z J n(n-2)
$$

Using this expresion in the analysis one should keep in mind that the MFA neglects the intersite correlation of fluctuation of the pseudospin operators what can lead to the error of order of $J=2 t^{2} /|U|$.

\section{ANALYTICAL RESULTS FOR WEAK AND STRONG $U$ CASES AT $T=0 \mathrm{~K}$}

The system of the selfconsistent equations (4, 51) can be approximately solved in the limiting cases of the weak and strong attraction limits and here we present these results:

A) The weak $-U$ case

Provided that the chemical potential is not located too close to van Hove singularity of the density of states (DOS) function $\mathcal{D}(\varepsilon)$, the gap parameter at $T=0$ can be obtained from equation (4) as: 


$$
\Delta=2 \sqrt{D_{+} D_{-}} \exp \left(\frac{I\left[\mathcal{C}^{\bar{\mu}}\right]}{2 \mathcal{D}(\bar{\mu})}\right) \exp \left(-\frac{1}{|U| \mathcal{D}(\bar{\mu})}\right)
$$

where: $D_{+}=D_{2}-\bar{\mu}, D_{-}=D_{1}+\bar{\mu}$ with $-D_{1}, D_{2}$ denoting lower and upper boundary of the band, respectively; $I\left[\mathcal{C}^{\bar{\mu}}\right]$ is a functional defined by

$$
I\left[f^{\bar{\mu}}\right]=\int_{-D_{1}}^{D_{2}} d \epsilon \operatorname{sign}(\epsilon-\bar{\mu}) f^{\bar{\mu}}(\epsilon)
$$

$\mathcal{C}^{\bar{\mu}}$ is a function of electron density, which depends on details of DOS and is defined by

$$
\mathcal{C}^{\bar{\mu}}(\epsilon)=\frac{\mathcal{D}(\epsilon)-\mathcal{D}(\bar{\mu})}{\epsilon-\bar{\mu}}
$$

In Eqs.(19),(21) the chemical potential may be approximated by its value in the normal state, $\bar{\mu}_{\mathrm{NO}}$ and it is determined by:

$$
n-1=-\int_{-D_{1}}^{D_{2}} d \epsilon \mathcal{D}(\epsilon) \operatorname{sign}\left(\epsilon-\bar{\mu}_{\mathrm{N}}\right)
$$

The critical temperature in this limit is given by:

$$
k_{\mathrm{B}} T_{\mathrm{C}} / \Delta \approx 1 / 1.76
$$

which is the well--known weak coupling BCS ratio.

Analogously, performing the weak coupling expansions of Eqs.(3, 5, 11) at $T=0$ one can find the analytical expressions determining $\lambda, \bar{\mu}_{\mathrm{SS}}, H_{\mathrm{C}}, \xi_{\mathrm{G}-\mathrm{L}}$ and $\kappa$. The London penetration depth is calculated as

$$
\frac{1}{\lambda^{2}(0)}=-K^{d i a}=-\frac{8 \pi e^{2}}{\hbar^{2} c^{2} a_{\perp}} \frac{1}{Z} E_{\mathrm{SS}}^{\mathrm{K}}(0)
$$

where 


$$
\begin{aligned}
E_{\mathrm{SS}}^{\mathrm{K}}(0)-E_{\mathrm{NO}}^{\mathrm{K}}(0) & =\frac{\Delta^{2}}{|U|}-\Delta^{2}\left\{\frac{E_{\mathrm{NO}}^{\mathrm{K}}-\bar{\mu} n}{4} \times\right. \\
& \times\left[\frac{1}{\left(D_{2}-\bar{\mu}\right)^{2}}+\frac{1}{\left(D_{1}+\bar{\mu}\right)^{2}}\right]+\frac{\bar{\mu}}{2\left(D_{2}-\bar{\mu}\right)^{2}} \\
& \left.+\mathcal{D}(\bar{\mu})+\frac{1}{2} I\left[\mathcal{C}^{\bar{\mu}}\right]-I\left[\mathcal{F}_{3}^{\bar{\mu}}\right]+\bar{\mu} I\left[\mathcal{G}_{3}^{\bar{\mu}}\right]\right\}
\end{aligned}
$$

where

$$
\begin{array}{rlrl}
\mathcal{G}_{n+1}^{\bar{\mu}}(\epsilon) & =\frac{\mathcal{G}_{n}^{\bar{\mu}}(\epsilon)-\mathcal{G}_{n}^{\bar{\mu}}(\bar{\mu})}{\epsilon-\bar{\mu}} \quad, & \mathcal{G}_{0}(\epsilon)=\int_{-D_{1}}^{\epsilon} d \epsilon^{\prime} \mathcal{D}\left(\epsilon^{\prime}\right) \epsilon^{\prime} \\
\mathcal{F}_{n+1}^{\bar{\mu}}(\epsilon)=\frac{\mathcal{F}_{n}^{\bar{\mu}}(\epsilon)-\mathcal{F}_{n}^{\bar{\mu}}(\bar{\mu})}{\epsilon-\bar{\mu}} \quad, & \mathcal{F}_{0}(\epsilon)=\int_{-D_{1}}^{\epsilon} d \epsilon^{\prime} \mathcal{D}\left(\epsilon^{\prime}\right)
\end{array}
$$

and $\bar{\mu}=\bar{\mu}_{\mathrm{NO}}$. Obviously, for $|U| / B \rightarrow 0 \quad E_{\mathrm{SS}}^{\mathrm{K}}(0)$ approaches the value of the band energy in the normal state.

In a case when we can neglect variation of $\mathcal{D}(\epsilon)$ and $D_{1}=D_{2}=D$ Eq.(25) reduces to

$$
E_{\mathrm{SS}}^{\mathrm{K}}(0)=-\mathcal{D}\left(\bar{\mu}_{\mathrm{NO}}\right)\left[D^{2}-\bar{\mu}_{\mathrm{NO}}^{2}+\frac{\Delta^{2}}{2}-\frac{\Delta^{2}}{|U| \mathcal{D}\left(\bar{\mu}_{\mathrm{NO}}\right)}\right]
$$

whereas $\bar{\mu}_{\mathrm{SS}}$ and $H_{\mathrm{C}}(0)$ are given by

$$
\bar{\mu}_{\mathrm{SS}}^{2}=\bar{\mu}_{\mathrm{NO}}^{2}\left(1+\frac{\Delta^{2}}{D^{2}-\bar{\mu}_{\mathrm{NO}}^{2}}\right)
$$

where $\bar{\mu}_{\mathrm{NO}}$ is given by Eq.(22), and

$$
\frac{H_{\mathrm{C}}^{2}(0)}{8 \pi}=\frac{E_{0}^{\mathrm{NO}}-E_{0}^{\mathrm{SS}}}{a_{\perp} a_{\|}^{2}} \approx \frac{1}{2} \frac{\Delta^{2} \mathcal{D}(\bar{\mu})}{a_{\perp} a_{\|}^{2}} .
$$

The weak coupling formulas for $\xi_{\mathrm{G}-\mathrm{L}}$ and $\kappa=\lambda / \xi_{\mathrm{G}-\mathrm{L}}$ follow directly from Eqs.(12), (24), (29). In the case of $\mathcal{D}_{1}(\bar{\mu})=0$ and $D_{1}=D_{2}=D$ their explicit forms are:

$$
\begin{aligned}
& \xi_{\mathrm{G}-\mathrm{L}}=\frac{a_{\|}}{2 \sqrt{2 Z}} \sqrt{-\frac{D^{2}-\bar{\mu}_{\mathrm{NO}}^{2}}{\Delta^{2}}+\frac{1}{2}-\frac{1}{|U| \mathcal{D}(\bar{\mu})} \approx \frac{a_{\|}}{2 \sqrt{2 Z}} \exp \left(\frac{1}{|U| \mathcal{D}(\bar{\mu})}\right)} \\
& \kappa=\frac{\Phi_{0} Z}{\pi \sqrt{\pi}} \frac{\sqrt{a_{\perp}}}{a_{\|}} \frac{\sqrt{\Delta^{2} \mathcal{D}\left(\bar{\mu}_{\mathrm{NO}}\right)}}{\left(D^{2}-\bar{\mu}_{\mathrm{NO}}^{2}+\frac{\Delta^{2}}{2}\right) \mathcal{D}\left(\bar{\mu}_{\mathrm{NO}}\right)-\frac{\Delta^{2}}{|U|}} \approx \\
& \approx \frac{\Phi_{0} Z}{\pi \sqrt{\pi}} \frac{\sqrt{a_{\perp}}}{a_{\|}} \frac{\exp \left(-1 /|U| \mathcal{D}\left(\bar{\mu}_{\mathrm{NO}}\right)\right)}{\sqrt{\left(D^{2}-\bar{\mu}_{\mathrm{NO}}^{2}\right) \mathcal{D}\left(\bar{\mu}_{\mathrm{NO}}\right)}}
\end{aligned}
$$


B) The strong $-U$ case

In this case the integrands in Eqs.(4,5) can be expanded in series with respect to $t / U$ and we have:

$$
\begin{aligned}
& x_{0}=\frac{\Delta}{|U|} \approx \frac{1}{2} \sqrt{1-\delta^{2}} \sqrt{1-4 L_{2}\left(\frac{t}{U}\right)^{2}+16 \delta L_{3}\left(\frac{t}{|U|}\right)^{3}}, \\
& \bar{\mu}_{\mathrm{SS}}=-\frac{|U|}{2} \delta\left(1+4 L_{2}(t / U)^{2}-4 L_{3}(3 \delta-1 / \delta)(t /|U|)^{3}\right),
\end{aligned}
$$

where $\delta=1-n, \bar{\mu}_{\mathrm{NO}}$ is given by (22) and the lattice sums $L_{n}$ represent the moments of DOS and are given by:

$$
L_{n}=\sum_{\vec{\delta}_{1} \ldots \vec{\delta}_{n}} \delta_{\vec{\delta}_{1}+\ldots \vec{\delta}_{n}, \overrightarrow{0}}
$$

One can note that odd moments vanish for the alternating (i.e. SQ and SC) lattices but not for the FCC ones. For alternating lattices $L_{2}=Z, L_{3}=0$ and for FCC lattice $L_{2}=12$ and $L_{3}=48$. The corresponding formula for $\lambda$ obtained by expanding of Eq.(3i) reads

$$
\frac{1}{\lambda^{2}}=\frac{8 \pi e^{2}}{\hbar^{2} c^{2} a_{\perp}} \frac{|U|}{Z}\left[2\left(1-\delta^{2}\right) L_{2}(t / U)^{2}-6 \delta\left(1-\delta^{2}\right) L_{3}(t /|U|)^{3}\right] .
$$

In the structures with asymmetric DOS (like FCC) the odd terms in $t / U$ from Eq.(35) are again nonzero. From (13) and (14) we obtain:

$$
\begin{gathered}
E_{0}^{\mathrm{NO}}=-\frac{1}{N} \sum_{\vec{k}} \epsilon_{\vec{k}} \operatorname{sign}\left(\epsilon-\bar{\mu}_{\mathrm{NO}}\right)-\frac{1}{4}|U| n^{2}, \\
E_{0}^{\mathrm{SS}}=-\frac{|U|}{2} n-n(2-n) L_{2} \frac{t^{2}}{|U|}+2 n(1-n)(2-n) L_{3} \frac{t^{3}}{U^{2}}, \\
\frac{H_{\mathrm{C}}^{2}}{8 \pi}=\frac{E_{0}^{\mathrm{NO}}-E_{0}^{\mathrm{SS}}}{a_{\|}^{2} a_{\perp}}=\frac{1}{a_{\|}^{2} a_{\perp}}\left\{\frac{|U|}{4} n(2-n)+n(2-n) \frac{z t^{2}}{|U|}\right\}+O\left(\frac{t^{3}}{U^{2}}\right),
\end{gathered}
$$




$$
\begin{gathered}
\xi_{\mathrm{G}-\mathrm{L}}=\frac{a_{\|}}{2}\left|\frac{t}{U}\right|\left[\frac{1}{4}+Z\left(\frac{t}{U}\right)^{2}\right]^{-1}, \\
\kappa=\frac{\Phi_{0}}{2 \pi \sqrt{\pi}} \frac{\sqrt{a_{\perp}}}{a_{\|}} \frac{1}{\sqrt{n(2-n)}} \frac{\sqrt{|U|^{3}}}{4 t^{2}} \sqrt{1+4 Z\left(\frac{t}{U}\right)^{2}} .
\end{gathered}
$$

It is worth while to compare the strong coupling results of HFA with the ones obtained for the effective pseudospin model Hamiltonian (16). Recently, the electromagnetic properties of that model have been analysed in [18,19] and below we only quote the expressions derived within MFA (the RPA treatment, taking into account quantum corrections, yields qualitatively similar results, except the low density limit [19]):

$$
\begin{gathered}
x_{0}=\frac{1}{2} \sqrt{n(2-n)}, \\
E_{0}^{\mathrm{SS}}=-\frac{1}{2}|U| n-\frac{1}{2} J_{0} n(2-n), \\
\frac{H_{\mathrm{C}}^{2}}{8 \pi}=\frac{1}{4} \frac{J_{0} n(2-n)}{a^{3}}, \\
\lambda^{-2}=\frac{2 \pi \bar{e}^{2}}{\hbar^{2} c^{2} a} \operatorname{Jn}(2-n),
\end{gathered}
$$

where $J_{0}=Z J, J=\frac{2 t^{2}}{|U|}$ and $\bar{e}=2 e, a_{\perp}=a_{\|}=a$,

$$
\begin{gathered}
\xi_{\mathrm{G}-\mathrm{L}}=\frac{a}{\sqrt{2 Z}}, \\
\kappa=\frac{\lambda}{\xi_{\mathrm{G}-\mathrm{L}}}=\frac{\Phi_{0} \sqrt{Z}}{2 \pi \sqrt{\pi} \sqrt{a \operatorname{Jn}(2-n)}},
\end{gathered}
$$

and $E_{0}^{\mathrm{NO}}$ is given by Eq.(18). Up to terms of order $t^{2} /|U|$ the HFA expressions for $x_{0}$ (Eq.(32)), $E_{0}^{\mathrm{SS}}$ (Eq.(37)) and $1 / \lambda^{2}$ (Eq.(35)) are equal to those given by Eqs.(41), (42) and (44). On the contrary the HFA results for $H_{\mathrm{C}}, \xi_{\mathrm{G}-\mathrm{L}}$ and $\kappa$ are qualitatively erroneous due to the great overestimation of $E_{0}^{\mathrm{NO}}$ by HFA in this limit (comp. Eq.(36) and Eq.(18)). Thus, 
to get realistic values of $H_{\mathrm{C}}, \xi_{\mathrm{G}-\mathrm{L}}$ and $\kappa$ for arbitrary $|U| / B$ one should combine the results obtained using $E_{0}^{\mathrm{NO}}$ given by Eq.(13) (reliable for $|U| / B<1$ ) with those obtained using $E_{0}^{\mathrm{NO}}$ given by Eq.(18) (reliable for $|U| / B>1$ ). This will be done in the next section, where we will also compare some of the predictions given above with the results of numerical solutions of Eqs.(3)-(5), (11)-(14).

\section{RESULTS OF NUMERICAL SOLUTION AND DISCUSSION.}

Figs.1a and 1b show the numerical plots of $T_{\mathrm{C}}(n), \Delta(n)$ and $\lambda^{-2}(n)$ for SC and FCC lattices, respectively (the corresponding plots for SQ lattice are given in Fig.1 of Ref. [20]), whereas Fig.1c presents the concentration dependence of $\Delta$ and $\lambda^{-2}(n)$ for the model rectangular $\mathcal{D}(\varepsilon)$ :

$$
\mathcal{D}(\varepsilon)=1 / B \text { for }-B / 2<\varepsilon<B / 2=D \text {, otherwise } 0 \text {, }
$$

where $B$ is the effective bandwidth. All these plots were made for the values of $U$ from the weak coupling regime $(|U| / B=0.1)$ where the effects of the density of states, $\mathcal{D}(\varepsilon)$, are most clearly seen. In this regime the superconducting critical temperature $T_{\mathrm{C}}$ and the $T=0$ gap parameter $\Delta$ increase rapidly with $\bar{\mu}$ approaching van Hove singularities in $\mathcal{D}(\varepsilon)$, in agreement with a modified BCS expression Eq.(19). This is the reason why $T_{\mathrm{C}}$ for $\mathrm{SQ}$ and SC lattices is symmetric with respect to $n=1$ and so strongly peaked for SQ lattice at $n=1$ while the position of $T_{\mathrm{C}}$ peak in the FCC structure moves with increase of $|U|$ from the vicinity of $n \approx 2$ toward $n=1$. For the FCC lattice the effect of density of states on the penetration depth consist mainly in $U$-dependent shift of the maximum of $1 / \lambda^{2}$ from $n=1$ towards $n<1$ (Fig.1b). The inverse squared penetration depth, being proportional to the

bandwidth for weak $-U$ decreases like $\sim Z t^{2} /|U|$ in the strong coupling limit. The plot of this quantity as a function of $n$ for a few values of $|U| / B$ is shown in Fig.2 (SQ lattice).

Examples of the evolution of the thermodynamic critical field $H_{\mathrm{C}}$ and of the correlation 
length $\xi_{\mathrm{G}-\mathrm{L}}$, with $|U|$ and $n$ are shown in Fig.3,4. Note the steep decrease of $\xi_{\mathrm{G}-\mathrm{L}}$ with $n$ in a relatively narrow range of $n$ values for the FCC lattice and substantial reduction of $\xi_{\mathrm{G}-\mathrm{L}}$ near $n=1$ for the SQ lattice (Fig. 5). These features can be understood on the basis of the $\Delta(n)$ behaviour presented in Figs 1,2.

The substantial variation of $\xi_{\mathrm{G}-\mathrm{L}}$ with $n$ for $|U| / B<1$ is largely due to the strong $n$ dependence of $H_{\mathrm{C}}^{2}$ which in the weak coupling limit is proportional to $\Delta^{2} \mathcal{D}(\bar{\mu})$ (cf. Eqs.(29), (30)).

With increasing $|U|$ the $n$-dependence of the correlation length is less pronounced and in the strong coupling regime, $|Z t / U| \ll 1, \xi_{\mathrm{G}-\mathrm{L}}$ calculated with in HFA goes like $\sim|Z t / U|$ being almost independent on $n$. However, for large $|U|$ the energy of the normal phase, entering (11), (12) is drastically overestimated in the HFA (comp. Sec. 3).

To get more realistic values of $H_{\mathrm{C}}$ and $\xi_{\mathrm{G}-\mathrm{L}}$ for $|U| / B>1$ we have made an estimation of $\xi_{\mathrm{G}-\mathrm{L}}$ and $H_{\mathrm{C}}$ exploiting perturbation approach in the calculation of the energy of the normal phase (Eq.(18)) and the numerical results for SQ lattice and $n=1$ are shown in Figs 3,4. In the $|U| / B \gg 1$ limit the analytical results for $H_{\mathrm{C}}, \xi_{\mathrm{G}-\mathrm{L}}$ and $\kappa$ can be obtained in this way by using Eqs. (18, 37, 35 and 33):

$$
\begin{gathered}
\frac{H_{\mathrm{C}}^{2}}{8 \pi}=\frac{E_{0}^{\mathrm{NO}}-E_{0}^{\mathrm{SS}}}{a_{\|}^{2} a_{\perp}}=\frac{1}{a_{\|}^{2} a_{\perp}}\left(\frac{1}{2} n(2-n) L_{2} \frac{t^{2}}{|U|}-2 n(1-n)(2-n) L_{3} \frac{t^{3}}{U^{2}}\right), \\
\xi_{\mathrm{G}-\mathrm{L}}=\frac{a_{\|}}{\sqrt{2 Z}} \sqrt{\frac{Z-3(1-n) L_{3} \frac{t}{|U|}}{Z-4(1-n) L_{3} \frac{t}{|U|}}}, \\
\kappa=\frac{\Phi_{0}}{2 \pi \sqrt{\pi}} \frac{Z \sqrt{a_{\perp}}}{a_{\|}} \frac{1}{\sqrt{n(2-n) \frac{2 t^{2}}{|U|}}} \frac{\sqrt{Z-4(1-n) L_{3} \frac{t}{|U|}}}{Z-3(1-n) L_{3} \frac{t}{|U|}} .
\end{gathered}
$$

They coincide (to the second order in $\frac{t}{|U|}$ ) with the MFA results for the pseudospin model (16) (cf. Eqs.(43), (45), (46)).

As we see from Figs $3 \mathrm{a}$ and 4 , for $|U| / B>1 H_{\mathrm{C}}$ decreases with increasing $|U| / B$ and for $|U| / t \gg 1, H_{\mathrm{C}}^{2} \sim t^{2} /|U|$, whereas $\xi_{\mathrm{G}-\mathrm{L}}$ tends to a fixed value $a / \sqrt{2 Z}$. 
Finally let us conclude the evolution of the Ginzburg ratio $\kappa=\lambda / \xi_{\mathrm{G}-\mathrm{L}}$ with $|U|$ and $n$. Concentration dependence of this quantity is plotted in Fig 6. Notice universal behaviours (i) $\kappa \sim n^{-1 / 2}$ for $n \ll 1$ (arbitrary $|U| / B$ ) and (ii) $\kappa \sim[n(2-n)]^{-1 / 2}$ for $|U| / t \gg 1$ (arbitrary $n$ ). In the weak-to-intermediate coupling regime $(|U| / B<1) \kappa(n)$ is not universal and strongly depends on the details of $\mathcal{D}(\epsilon)$. From the equation $\lambda / \xi_{\mathrm{G}-\mathrm{L}}=1 / \sqrt{2}$ one can estimate the boundaries between local and nonlocal electromagnetic behaviour in the considered model 16, 17. In Fig. 7 we plot this boundary in the $|U| / B-n$ parameter space for fixed value of $t$ and $a$. In the low concentration limits $(|n-1| \sim 1)$ the local behaviour extends up to very small values of $|U| / B$.

The high- $T_{\mathrm{C}}$ systems (not only the cuprates but also the barium bismuthates and the fullerides) are extreme type II superconductors with local electromagnetic behaviour and with very large Ginzburg ratio estimated to be of order $10^{2}$ [3, 21]. Thus, from the equality $\kappa=10^{2}$ one can estimate the values of $|U| / B$ and $n$ which could be reliable for these materials. Examples of such estimations are given in Fig. 8 and they indicate that best agreement can be obtained for intermediate values of the local attraction.

In order to make some more detailed comparisons with experiment we have evaluated the plots $T_{\mathrm{C}} / T_{\mathrm{C}}^{\mathrm{m}}$ vs. $\left(\lambda^{\mathrm{m}} / \lambda\right)^{2}$, where $T_{\mathrm{C}}^{\mathrm{m}}$ denotes the maximum critical temperature in the $0<n<2$ range and $\lambda^{\mathrm{m}}$ corresponds to $T_{\mathrm{C}}^{\mathrm{m}}$. For a given $|U| / B$ the data corresponding to $\mathrm{SQ}$ and SC lattices represent the one-valued function (see Figs.9ab), whereas the ones for the FCC lattice (see Fig.9c) exhibit substantial hysteresis - the behaviour for the FCC structure being due to the difference in positions of $T_{\mathrm{C}}(n)$ and $1 / \lambda^{2}(n)$ maxima seen in Fig.1b. At present the available experimental data [4,22,1,2] correspond mainly to SQ lattices (cuprates) and alternating cubic lattices (Chevrel phases, doped $\mathrm{BaBiO}_{3}$ ) and except of the overdoped cases they compare well with the present plots for these lattices in the intermediate coupling range $(|U| / B \leq 1)$.

The experimentally found deviations from the $T_{\mathrm{C}} / T_{\mathrm{C}}^{\mathrm{m}}$ vs. $\left(\lambda^{\mathrm{m}} / \lambda\right)^{2}$ universal dependence 
in the strongly overdoped regime can be explained either by the asymmetry in $\mathcal{D}(\varepsilon)(\mathrm{cf}$ Fig 9c) due to further neighbour hopping in SC and SQ structures or by the effects of intersite Coulomb interactions [18], which can be different for various families of materials.

Very recently Locquet [23] have reported the penetration depth measurements for $\mathrm{La}_{2-\mathrm{x}}$

$\mathrm{Sr}_{\mathrm{x}} \mathrm{CuO}_{4}$ films as a function of doping, extending for the first time in the range from heavily underdoped to heavily overdoped. As we see from Fig.10 the theoretical plots $\lambda(n) / \lambda(n=1)$ for our simple effective model for SQ lattice fit surprisingly well these experimental data.

\section{ACKNOWLEDGMENTS}

This work has financial supports from K.B.N. Poland, projects 2 P03B 104 11, 2P03B05709.

We are grateful to Prof. R. Micnas for very helpful discussion. One of us (W.R.C.) wants to thank to the Center de Recherches sur les Très Basses Températures (CRTBT) of the Centre National de la Recherche Scientifique in Grenoble for hospitality and to the UESCO/ICSU/TWAS for a grant Nr. SC/RP 206.770.5 which made possible his stay in the CRTBT during the course of this work. Thanks are also due to the French-Polish Scientific and Technological Cooperation Joint Project for 1996, Proj. Nr. 6448. 


\section{APPENDIX A}

For rectangular DOS defined by (47). one can derive from Eqs.(10-12) analytical expressions determining $\lambda, H_{\mathrm{C}}, \xi_{\mathrm{G}-\mathrm{L}}$ and $\kappa$ as a function of $n$ and $|U| / D$ at $T=0$.

In particular, from Eqs.(47) taken at $T=0$ one obtains 15

$$
\begin{gathered}
\Delta=\sqrt{n(2-n)} D / \sinh \frac{2 D}{|U|}, \\
\bar{\mu}_{\mathrm{SS}}=(n-1) D \operatorname{coth} \frac{2 D}{|U|}, \\
\bar{\mu}_{\mathrm{NO}}=(n-1) D, \\
E_{0}^{\mathrm{NO}}=-\frac{D}{2} n(2-n)-\frac{|U|}{4} n^{2}, \\
E_{0}^{\mathrm{SS}}=-\frac{D}{2} n(2-n) \operatorname{coth} \frac{2 D}{|U|}-\frac{|U|}{4} n^{2},
\end{gathered}
$$

whereas $1 / \lambda^{2}(0)=-K^{\text {dia }}$ calculated from Eqs.(3) and (4), (5) is:

$$
\frac{1}{\lambda_{L}^{2}}=\frac{4 \pi e^{2} t}{\hbar^{2} c^{2} a_{\perp}} n(2-n)\left[\operatorname{coth} \frac{2 D}{|U|}-\frac{2 D}{|U|}\left(\sinh \frac{2 D}{|U|}\right)^{-2}\right]
$$

Taking into account Eqs.(A4)-(A6) in Eqs.(11) and (12) one finds the following expressions for $H_{\mathrm{C}}, \xi_{\mathrm{G}-\mathrm{L}}$ and $\kappa$ :

$$
\begin{gathered}
\frac{H_{\mathrm{C}}^{2}}{8 \pi}=\left(\frac{\Delta_{E}}{a_{\|}^{2}(\AA) a_{\perp}(\AA)}\right), \\
\Delta_{E}=E_{0}^{\mathrm{NO}}-E_{0}^{\mathrm{SS}}=\frac{D n(2-n)}{2}\left[\operatorname{coth} \frac{2 D}{|U|}-1\right], \\
\xi_{\mathrm{G}-\mathrm{L}}=\frac{a_{\|}}{2 \sqrt{2 Z}} \sqrt{\frac{\operatorname{coth} \frac{2 D}{|U|}-\frac{2 D}{|U|}\left(\sinh \frac{2 D}{|U|}\right)^{-2}}{\left[\operatorname{coth} \frac{2 D}{|U|}-1\right]}},
\end{gathered}
$$

where $D=Z t$. 


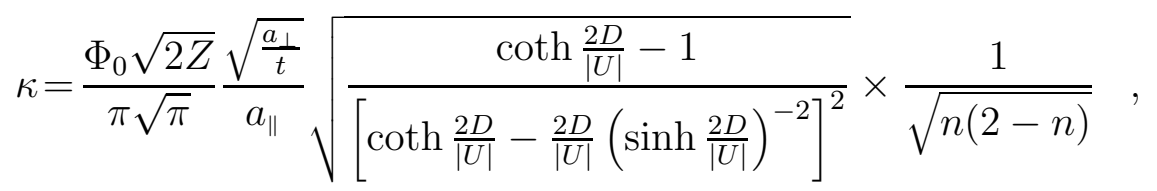

In the limits (i) $|U| / D \ll 1$ and (ii) $|U| / D \gg 1$ the above equations take the form: for (i)

$$
\begin{gathered}
\frac{1}{\lambda_{L}^{2}}=\frac{4 \pi e^{2} t}{\hbar^{2} c^{2} a_{\perp}} n(2-n)\left[1-\frac{8 D}{|U|} \exp \left(-\frac{4 D}{|U|}\right)\right], \\
\Delta_{E}=D n(2-n) \exp \left(-\frac{4 D}{|U|}\right), \\
\xi_{\mathrm{G}-\mathrm{L}}=\frac{a_{\|}}{2 \sqrt{2 Z}} \exp \left(\frac{2 D}{|U|}\right) \\
\kappa=\frac{\Phi_{0} \sqrt{2 Z}}{\pi \sqrt{\pi}} \frac{\sqrt{\frac{a_{\perp}}{t}}}{a_{\|}} \frac{\exp \left(\frac{2 D}{|U|}\right)}{\sqrt{n(2-n)}}
\end{gathered}
$$

for (ii):

$$
\begin{gathered}
\frac{1}{\lambda^{2}(0)}=\frac{16 \pi e^{2}}{\hbar^{2} c^{2} a_{\perp}} \frac{Z}{3} n(2-n) \frac{t^{2}}{|U|}, \\
\Delta_{E}=\frac{D n(2-n)}{2}\left[\frac{|U|}{2 D}+\frac{2 D}{3|U|}-1\right], \\
\xi_{\mathrm{G}-\mathrm{L}}=\frac{a_{\|} t}{|U|},
\end{gathered}
$$

For $|U| / D \gg 1$ the results for $H_{\mathrm{C}}$ (and consequently also for $\xi_{\mathrm{G}-\mathrm{L}}$ and $\kappa$ ) are unrealistic as $E_{0}^{\mathrm{NO}}$ in this limit largely overestimated by MFA. As we have quoted in Sec. 2, one can correct these results by using Eq.(18) instead of (A4) in the calculation of $H_{\mathrm{C}}$. 
Mailing address: Wojciech R. Czart, Institute of Physics, A. Mickiewicz University, Umultowska 85, 61-614 Poznań, Poland, e-mail: czart@phys.amu.edu.pl. 


\section{REFERENCES}

[1] Y. J. Uemura et al. Nature 352, 605 (1991); Phys. Rev. Lett. 66, 2665 (1991).

[2] T. Schneider, K. Keller, Phys. Rev. Lett. 69, 3374 (1992); Int. J. Mod. Phys. B 8, 487 (1994).

[3] R. Micnas, R. Ranninger, S. Robaszkiewicz, Rev. Mod. Phys. 62, 113 (1990).

[4] T. Schneider, H. Beck, D. Bormann, T. Meintrup, S. Schafroth, A. Schmidt, Physica C 216, 432 (1993).

[5] J. A. Wilson, J. Phys. C 20 L 911(1987); ibid.21, 2067 (1988); idem, Physica C 233, 322 (1994).

[6] N. F. Mott, Physica A 200, 127 (1993);

idem, J. Phys. Condens. Matt. 5, 3487 (1993).

[7] A. Taraphder et al. Phys. Rev. B 52, 1368 (1995); Europhys. Lett. 21, 79 (1993).

[8] C. M. Varma, Phys. Rev. Lett. 61, 2713 (1989).

[9] S. K. Sarker, Phys. Rev. B 49, 12047 (1994).

[10] F. C. Zhang, M. Ogata and T. M. Rice, Phys. Rev. Lett. 67, 3452 (1991).

[11] S. Chakravarty, S. Kivelson, Europhys. Lett. 16, 751 (1991).

[12] S. Chakravarty, M. P. Gelfand, S. Kivelson, Science 254, 970 (1991).

[13] J. A. Wilson, Physica C 182, 1 (1991).

[14] T. Kostyrko and R. Micnas Phys. Rev. B 46, 11025 (1992).

[15] S. Robaszkiewicz, R. Micnas, K. A. Chao, Phys. Rev. B 24, 4018 (1981).

[16] A. L. Fetter, J. D. Walecka, Quantum Theory of Many-Particle Systems, McGraw-Hill, 
New York, (1971).

[17] D. J. Scalapino, S. R. White, S. Zhang, Phys. Rev. B 47, 7995 (1993); T. Kostyrko, R. Micnas, K. A. Chao, Phys. Rev. B 49, 6158 (1994).

[18] S. Robaszkiewicz, T. Kostyrko, R. Micnas, Physica C 234-240, 1827 (1994).

[19] R. Micnas, S. Robaszkiewicz, T. Kostyrko Phys. Rev. B 52, 6863 (1995).

[20] W. Czart, T. Kostyrko and S. Robaszkiewicz, J. Mag. Mag. Mat. 140-144, 2059 (1995).

[21] K. Holczer, Int. J. Mod. Phys. B 6, 3967 (1992).

[22] Ch. Niedermayer, C. Bernhard, U. Binninger, H. Glücker, J. L. Tallon, E. J. Ansaldo, J. I. Budnick, Phys. Rev. Lett. 71, 1764 (1993).

[23] J. P. Locquet et al. Phys. Rev. Lett. (1995) preprint; T. Schneider in "Polarons and Bipolarons in High $T_{\mathrm{C}}$ Superconductors and Related Materials", (Eds. E. K. H. Salje, A. S. Alexandrov, W. Y. Liang), Cambridge Univ. Press 1995), p. 258. 


\section{FIGURE CAPTIONS}

Fig. 1 Concentration dependence of $T_{\mathrm{C}}$, the gap parameter $\Delta$ and $1 / \lambda^{2}$ at $T=0 \mathrm{~K}$ : $|U| / B=0.1$ ( $B=$ bandwidth); a) SC lattice, b) FCC lattice, c) model rectangular density of states. (here: $e^{2} / \hbar^{2} c^{2} a=1, a$-lattice constant, $B$-bandwidth).

Fig. 2 Concentration dependence of $1 / \lambda^{2}$ at $T=0 \mathrm{~K}$ for different values of $U / B$ for SQ lattice.

Fig. 3a Reduced square value of thermodynamic critical field $H_{\mathrm{C}}$ at $T=0\left(h_{\mathrm{C}}^{2} / B=\right.$ $\left.H_{\mathrm{C}}^{2} a^{2} /(8 \pi B)\right)$ as a function of $|U| / B$ for $n=1$ for SQ lattice. HFA (o), perturbation theory: $0^{\text {th }}$ order (obtained using Eqs.(11, 18, 42) for $\left.J=0\right)(*), 2^{\text {nd }}$ order (obtained using Eqs. (11, 18, 42) for $J \neq 0)(+)$.

Fig. $3 \mathbf{b} h_{\mathrm{C}}^{2} / B$ vs $n$ in $T=0$ plotted for increasing values of $|U| / B$ in HFA for SQ lattice. (numbers to the curves).

Fig. 4 The G-L correlation length $\xi_{\mathrm{G}-\mathrm{L}}$ as a function of $|U| / B$ for $n=1$ for SQ lattice. HFA (solid line), perturbation theory: $0^{\text {th }}$ order (obtained by putting $J=0$ in Eqs. (12, 18, (42)) (dotted line), $2^{\text {nd }}$ order (obtained using Eqs.(12,18, 12) for $J \neq 0$ ) (dashed line), $T=0$ $\left(\xi_{0}=\frac{a_{\|}}{\sqrt{2}}\right)$.

Fig. $5 \xi_{\mathrm{G}-\mathrm{L}}(0)$ vs $n$ for SQ (full circles) and FCC (triangles) lattices: $|U| / B=0.3$.

Fig. 6 Concentration dependence of $\kappa / \kappa_{0}\left(\kappa=\lambda / \xi_{\mathrm{G}-\mathrm{L}}\right.$ at $\left.T=0, \kappa_{0}=\kappa(n=1)\right)$ calculated for SQ lattice for several fixed values of $|U| / B$ (numbers to the curves). The corresponding plot obtained for a model rectangular DOS for arbitrary $|U| / B$ is shown by a dashed line.

Fig. 7 The boundary between local and nonlocal electromagnetic behaviour in the $|U| / B$

- $n$ parameter space calculated from the equation $\kappa=1 / \sqrt{2}$ for $t=0.15 \mathrm{eV}, a_{\|}=3.85 \AA$ and 
$a_{\perp}=7.7 \AA$ for rectangular DOS.

Fig. 8 The values of $|U| / B$ for the attractive Hubbard model which provide the Ginzburg ratio $\kappa=10^{2}$. The plots as a function of $n$ for SQ lattice, $t=0.15 \mathrm{eV}, a_{\|}=3.85 \AA$, $a_{\perp}=7.7 \AA$ (solid line) and for rectangular DOS $t=0.15 \mathrm{eV}, a=7.7 \AA$ (dashed line).

Fig. $9 \frac{T_{\mathrm{C}}}{T_{\mathrm{C}}^{\mathrm{m}}}$ vs. $\left(\frac{\lambda^{\mathrm{m}}}{\lambda}\right)^{2}$ at $T=0 \mathrm{~K}$ a) for the SQ lattice, b) SC lattice $\frac{|U|}{B}=2(-), \frac{|U|}{B}=0.5(\cdots)$, $\frac{|U|}{B}=0.2(--)$ and c) for FCC lattice: $\frac{|U|}{B}=5(--), \frac{|U|}{B}=1(--), \frac{|U|}{B}=0.2(-)$. $\lambda^{\mathrm{m}}$ corresponds to the maximum transition temperature $T_{\mathrm{C}}^{\mathrm{m}}$. (o)- $-\mathrm{Tl}_{2} \mathrm{Ba}_{2} \mathrm{Ca}_{2} \mathrm{Cu}_{3} \mathrm{O}_{10}, \mathrm{Tl}_{0.5} \mathrm{~Pb}_{0.5} \mathrm{Sr}_{2}$ $\mathrm{Ca}_{2} \mathrm{Cu}_{3} \mathrm{O}_{9}, \mathrm{Bi}_{2-\mathrm{x}} \mathrm{Pb}_{\mathrm{x}} \mathrm{Sr}_{2} \mathrm{Ca}_{2} \mathrm{Cu}_{3} \mathrm{O}_{16} ;(\diamond)-\mathrm{Y}_{1-\mathrm{x}} \mathrm{Pr}_{\mathrm{x}} \mathrm{Ba}_{2} \mathrm{Cu}_{3} \mathrm{O}_{6.97} ;(\triangle)-\mathrm{YBa}_{22} \mathrm{Cu}_{3} \mathrm{O}_{\mathrm{x}} ;(\nabla)-\mathrm{La}_{2-\mathrm{x}}$ $\mathrm{Sr}_{\mathrm{x}} \mathrm{CuO}_{4} ;(\star)-\mathrm{Bi}_{2} \mathrm{Sr}_{2} \mathrm{Ca}_{1-\mathrm{x}} \mathrm{Y}_{\mathrm{x}} \mathrm{Cu}_{2} \mathrm{O}_{8+\delta} ;(\sqcap)-\mathrm{LaMo}_{6} \mathrm{Se}_{2}, \mathrm{PbMo}_{6} \mathrm{~S}_{8}, \mathrm{SnMo}_{6} \mathrm{~S}_{4} \mathrm{Se}_{4}, \mathrm{SnMo}_{6} \mathrm{~S}_{7} \mathrm{Se}$, $\mathrm{SnMo}_{6} \mathrm{~S}_{1} \mathrm{Se}_{7}, \mathrm{LaMo}_{8} \mathrm{~S}_{8}, \mathrm{PbMo}_{6} \mathrm{~S}_{4} \mathrm{Se}_{4} ;(\oplus)-\mathrm{Tl}_{2} \mathrm{Ba}_{2} \mathrm{CuO}_{6+\delta}$. Experimental data taken from [22,1].

Fig. $10 \frac{\lambda(n)}{\lambda(n=1)}$ at $T=0 \mathrm{~K}$ for SQ lattice $\left(\frac{|U|}{B}=0.1(*), \frac{|U|}{B}=0.5(\circ)\right)$, compared with experimental results (full squares) [23] $\mathrm{La}_{2-\mathrm{x}} \mathrm{Sr}_{\mathrm{x}} \mathrm{CuO}_{4}$ films ( $-39 \mathrm{~nm}$ ) (squares), $\lambda_{0}=\lambda(x=0.159)=$ $[6700 \pm 500] \AA$. The results for a model square density of states almost coincide with the curve (o) for arbitrary $|U / B|$. 
This figure "fig_1a.gif" is available in "gif" format from: http://arxiv.org/ps/cond-mat/9701046v1 
This figure "fig_1b.gif" is available in "gif" format from: http://arxiv.org/ps/cond-mat/9701046v1 
This figure "fig_1c.gif" is available in "gif" format from: http://arxiv.org/ps/cond-mat/9701046v1 
This figure "fig_2.gif" is available in "gif" format from: http://arxiv.org/ps/cond-mat/9701046v1 
This figure "fig_3a.gif" is available in "gif" format from: http://arxiv.org/ps/cond-mat/9701046v1 
This figure "fig_3b.gif" is available in "gif" format from: http://arxiv.org/ps/cond-mat/9701046v1 
This figure "fig_4.gif" is available in "gif" format from: http://arxiv.org/ps/cond-mat/9701046v1 
This figure "fig_5.gif" is available in "gif" format from: http://arxiv.org/ps/cond-mat/9701046v1 
This figure "fig_6.gif" is available in "gif" format from: http://arxiv.org/ps/cond-mat/9701046v1 
This figure "fig_7.gif" is available in "gif" format from: http://arxiv.org/ps/cond-mat/9701046v1 
This figure "fig_8.gif" is available in "gif" format from: http://arxiv.org/ps/cond-mat/9701046v1 
This figure "fig_9a.gif" is available in "gif" format from: http://arxiv.org/ps/cond-mat/9701046v1 
This figure "fig_9b.gif" is available in "gif" format from: http://arxiv.org/ps/cond-mat/9701046v1 
This figure "fig_9c.gif" is available in "gif" format from: http://arxiv.org/ps/cond-mat/9701046v1 
This figure "fig_10.gif" is available in "gif" format from: http://arxiv.org/ps/cond-mat/9701046v1 